\title{
A LIOUVILLE THEOREM ON COMPLETE NON-KÄHLER MANIFOLDS
}

\author{
YUANG LI, CHUANJING ZHANG AND XI ZHANG
}

\begin{abstract}
In this paper, we prove a Liouville theorem for holomorphic functions on a class of complete Gauduchon manifolds. This generalizes a result of Yau for complete Kähler manifolds to the complete non-Kähler case.
\end{abstract}

\section{INTRODUCTION}

Let $(M, g)$ be a complete Riemannian manifold and $\Delta$ be the Beltrami-Laplacian of the Riemannian metric. In [18], Yau studied the equation

$$
\Delta \log u=f,
$$

and proved that if $0<\int_{M} f d v_{g} \leq \infty$ or if $f \equiv 0$, then the equation (1.1) has no nonconstant $L^{p}$-solutions for $0<p<\infty$. As an application, Yau obtained the following $L^{p}$ Liouville theorem.

Theorem 1.1 ([18], Theorem 4). Let $(M, \omega)$ be a complete Kähler manifold. Then there is no non-constant $L^{p}$ holomorphic functions for $p>0$.

It should be pointed out that, under the assumption that $(M, \omega)$ has sectional curvature of the same sign, Greene-Wu ([7]) got some lower bound for the $L^{p}$ integral of holomorphic functions. In this paper, we want to study the $L^{p}$ Liouville theorem of holomorphic functions for some complete non-Kähler manifolds.

From now on, let $(M, g)$ be a complete Hermitian manifold of complex dimension $n$ and $\omega$ the associated $(1,1)$-form. The metirc $g$ is called Gauduchon if $\omega$ satisfies $\partial \bar{\partial} \omega^{n-1}=0$. In [6], Gauduchon proved that, when $(M, g)$ is compact, there must exist a Gauduchon metric $g_{0}$ in the conformal class of $g$. It is interesting to generalize some geometric results from Kähler manifolds to Gauduchon manifolds, for example, the Donaldson-UhlenbeckYau theorem ([14], 4], [17]) is valid for Gauduchon manifolds (see [2, 10, 12, 11]).

Let $\varphi$ be a holomorphic function on $M$, it is easy to check that $\partial \bar{\partial} \log |\varphi| \equiv 0$. Following Yau's argument in [18], we consider the following equation

$$
\widetilde{\Delta} \log u=\sqrt{-1} \Lambda_{\omega} \partial \bar{\partial} \log u=f,
$$

2010 Mathematics Subject Classification: 53C55; 53C07; 58E20.

Key words and phrases: holomorphic function; Gauduchon manifold; Liouville theorem

The authors were supported in part by NSF in China, No. 11625106, 11571332 and 11721101. 
where $\Lambda_{\omega}$ denotes the contraction with $\omega, u \geq 0$ and the Hausdorff measure of $\{x \in$ $M \mid u(x)=0\}$ is zero. It is well known that the difference of two Laplacian is given by a first order differential operator as follows

$$
\left(\widetilde{\Delta}-\frac{1}{2} \Delta\right) \psi=V \cdot \nabla \psi
$$

where $V$ is a vector field on $M$. In the non-Kähler case, we should handle the first order term, the key is to control the vector field $V$ in order to use the Stokes' theorem which was prove by Yau (Lemma in [18]). In fact, we can prove the following theorem.

Theorem 1.2. Let $(M, g)$ be a complete Gauduchon manifold of complex dimension $n$ with $\left|d\left(\omega^{n-1}\right)\right| \in L^{\infty}(M)$. Suppose $f$ is bounded from below by a constant and is Lebesgue integrable with $0<\int_{M} f d v_{g} \leq \infty$ or $f \equiv 0$. Then there is no non-constant $L^{p}$ smooth solution of the equation (1.2) for $0<p<\infty$.

As an application, we obtain the following Liouville theorem.

Theorem 1.3. Let $(M, g)$ be a complete Gauduchon manifold of complex dimension $n$ with $\left|d\left(\omega^{n-1}\right)\right| \in L^{\infty}(M)$. Then there is no non-constant $L^{p}$ holomorphic functions for $0<p<\infty$.

Remark: Let $\left(M_{1}, g_{1}\right)$ be a compact Gauduchon manifold and $\left(M_{2}, g_{2}\right)$ be a complete Kähler manifold, it is easy to check that the product Riemannian manifold $\left(M_{1} \times M_{2}, g_{1} \times\right.$ $\left.g_{2}\right)$ is a Gauduchon manifold satisfying the assumption in Theorem 1.2.

This paper is organized as follows. In Section 2, we give a proof of Theorems 1.2. In Section 3, we consider a vanishing theorem on Higgs bundles over complete non-Kähler manifolds.

\section{Proof of Theorem 1.2}

In [18], Yau established the following generalized Stokes theorem which is an extension of Gaffney's result ([5]).

Lemma 2.1 ([18, Lemma ]). Let $(N, h)$ be a complete Riemannian manifold and $\eta$ be a smooth integrable $(\operatorname{dim} N-1)$-form defined on $M$. Then there exists a sequence of domains $B_{i}$ in $N$ such that $N=\cup_{i} B_{i}, B_{i} \subset B_{i+1}$ and $\lim _{i \rightarrow+\infty} \int_{B_{i}} d \eta=0$.

Let $\epsilon>0$, as that in [18], we set $v_{\epsilon}=(u+\epsilon)^{\frac{1}{2}}$. Form direct computations, it follows that

$$
\sqrt{-1} \Lambda_{\omega} \partial \bar{\partial} \log v_{\epsilon}=\sqrt{-1} \Lambda_{\omega} \partial \bar{\partial} \log u \cdot \frac{u}{2(u+\epsilon)}+\frac{\epsilon|\partial u|^{2}}{2 u(u+\epsilon)}
$$

and

$$
\sqrt{-1} \Lambda_{\omega} \partial \bar{\partial} \log v_{\epsilon}=\sqrt{-1} \Lambda_{\omega} \frac{\partial \bar{\partial} v_{\epsilon}}{v_{\epsilon}}-\sqrt{-1} \Lambda_{\omega} \frac{\partial v_{\epsilon} \wedge \bar{\partial} v_{\epsilon}}{v_{\epsilon}^{2}}
$$

Thus we have

$$
v_{\epsilon} \sqrt{-1} \Lambda_{\omega} \partial \bar{\partial} v_{\epsilon}=\left|\partial v_{\epsilon}\right|^{2}+\frac{\sqrt{-1} \Lambda_{\omega} \partial \bar{\partial} \log u}{2} \cdot u+\frac{\epsilon|\partial u|^{2}}{2 u}
$$


Let $0<R_{1}<R_{2}$ be any positive numbers. Fix a point $x_{0} \in M$, and choose a nonnegative cut-off function $\varphi$ satisfying

$$
\varphi(x)= \begin{cases}1, & x \in B_{x_{0}}\left(R_{1}\right), \\ 0, & x \in M \backslash B_{x_{0}}\left(R_{2}\right),\end{cases}
$$

$0 \leq \varphi \leq 1$ and $|d \varphi|_{\omega} \leq \frac{C}{R_{2}-R_{1}}$, where $C$ is a positive constant and $B_{x_{0}}(r)$ is the geodesic ball centered at $x_{0}$ with radius $r$. According to the Stokes formula and the condition $\partial \bar{\partial}\left(\omega^{n-1}\right)=0$, one can check that

$$
\begin{aligned}
& \int_{B\left(R_{2}\right)} \varphi^{2} v_{\epsilon} \sqrt{-1} \Lambda_{\omega} \partial \bar{\partial} v_{\epsilon} \frac{\omega^{n}}{n !} \\
= & \int_{M} \varphi^{2} v_{\epsilon} \sqrt{-1} \partial \bar{\partial} v_{\epsilon} \wedge \frac{\omega^{n-1}}{(n-1) !} \\
= & \int_{M} \partial\left(\varphi^{2} v_{\epsilon} \sqrt{-1 \partial} v_{\epsilon} \wedge \frac{\omega^{n-1}}{(n-1) !}\right)-\sqrt{-1} \partial \varphi^{2} \wedge v_{\epsilon} \bar{\partial} v_{\epsilon} \wedge \frac{\omega^{n-1}}{(n-1) !} \\
& -\sqrt{-1} \varphi^{2} \partial v_{\epsilon} \wedge \bar{\partial} v_{\epsilon} \wedge \frac{\omega^{n-1}}{(n-1) !}+\varphi^{2} v_{\epsilon} \sqrt{-1 \partial} v_{\epsilon} \wedge \partial \frac{\omega^{n-1}}{(n-1) !} \\
= & -2 \int_{M} \varphi v_{\epsilon}\left\langle\partial \varphi, \bar{\partial} v_{\epsilon}\right\rangle_{g} \frac{\omega^{n}}{n !}-\int_{M} \varphi^{2}\left|\partial v_{\epsilon}\right|^{2} \frac{\omega^{n}}{n !}+\frac{1}{2} \int_{M} \varphi^{2} \sqrt{-1 \partial} v_{\epsilon}{ }^{2} \wedge \partial \frac{\omega^{n-1}}{(n-1) !} \\
= & -2 \int_{M} \varphi v_{\epsilon}\left\langle\partial \varphi, \bar{\partial} v_{\epsilon}\right\rangle_{g} \frac{\omega^{n}}{n !}-\int_{M} \varphi^{2}\left|\partial v_{\epsilon}\right|^{2} \frac{\omega^{n}}{n !} \\
& +\frac{1}{2} \int_{M} \sqrt{-1 \partial}\left(\varphi^{2} v_{\epsilon}{ }^{2} \partial \frac{\omega^{n-1}}{(n-1) !}\right)-\sqrt{-1} v_{\epsilon}^{2} \bar{\partial} \varphi^{2} \wedge \partial \frac{\omega^{n-1}}{(n-1) !} \\
\leq & \int_{M}\left(v_{\epsilon}^{2}|\partial \varphi|^{2}+C^{\star} v_{\epsilon}{ }^{2} \varphi|\partial \varphi|\right) \frac{\omega^{n}}{n !},
\end{aligned}
$$

where we have used the condition that $\left|d\left(\omega^{n-1}\right)\right| \in L^{\infty}(M)$ in the last inequality .

Combining (1.2), (2.3) and (2.5), we obtain

$$
\begin{aligned}
& \frac{1}{4} \int_{M} \varphi^{2} \frac{|\partial u|^{2}}{u+\epsilon} \frac{\omega^{n}}{n !} \\
= & \int_{M} \varphi^{2}\left|\partial v_{\epsilon}\right|^{2} \frac{\omega^{n}}{n !} \\
\leq & \int_{M} v_{\epsilon}{ }^{2}\left(|\partial \varphi|^{2}+C^{\star} \varphi|\partial \varphi|\right) \frac{\omega^{n}}{n !}-\int_{M} \frac{f \varphi^{2} u}{2} \\
\leq & \left(\frac{C^{2}}{\left(R_{1}-R_{2}\right)^{2}}+\frac{C^{\star} C}{\left(R_{2}-R_{1}\right)}\right) \int_{B\left(R_{2}\right)} v_{\epsilon}{ }^{2} \frac{\omega^{n}}{n !}-\frac{1}{2} \int_{M} f \varphi^{2} u \frac{\omega^{n}}{n !},
\end{aligned}
$$

and

$$
\begin{aligned}
& \frac{1}{4} \int_{B\left(R_{2}\right)} \varphi^{2} \frac{|\partial u|^{2}}{u} \frac{\omega^{n}}{n !} \\
\leq & \left(\frac{C^{2}}{\left(R_{1}-R_{2}\right)^{2}}+\frac{C^{\star} C}{\left(R_{2}-R_{1}\right)}\right) \int_{B\left(R_{2}\right)} u^{2} \frac{\omega^{n}}{n !}-\frac{1}{2} \int_{B\left(R_{2}\right)} f \varphi^{2} u \frac{\omega^{n}}{n !} .
\end{aligned}
$$


Let $R_{1}=2 R_{2} \rightarrow \infty$, we deduce

$$
\int_{M} \frac{|d u|^{2}}{u} \frac{\omega^{n}}{n !}<\infty
$$

and then

$$
\int_{M}|d u| \frac{\omega^{n}}{n !} \leq\left(\int_{M} \frac{|d u|^{2}}{u} \frac{\omega^{n}}{n !}\right)^{\frac{1}{2}}\left(\int_{M} u \frac{\omega^{n}}{n !}\right)^{\frac{1}{2}}<\infty .
$$

On the other hand, it is easy to see that

$$
\sqrt{-1} \Lambda_{\omega} \partial \bar{\partial} \log u_{\epsilon}=\sqrt{-1} \Lambda_{\omega} \partial \bar{\partial} \log u \cdot \frac{u}{u+\epsilon}+\frac{\epsilon}{u(u+\epsilon)^{2}}|\partial u|^{2}
$$

and

$$
\int_{M}\left|\partial \log u_{\epsilon}\right| \frac{\omega^{n}}{n !}=\int_{M} \frac{|\partial u|}{u+\epsilon} \frac{\omega^{n}}{n !} \leq \int_{M} \frac{|d u|}{\epsilon} \frac{\omega^{n}}{n !}<\infty
$$

for any $\epsilon>0$, where $u_{\epsilon}=u+\epsilon$. Using the Stokes formula and the condition $\partial \bar{\partial}\left(\omega^{n-1}\right)=0$ again, we derive

$$
\begin{aligned}
& \sqrt{-1} \Lambda_{\omega} \partial \bar{\partial} \log u_{\epsilon} \frac{\omega^{n}}{n !} \\
= & \sqrt{-1} \partial\left(\bar{\partial} \log u_{\epsilon} \wedge \frac{\omega^{n-1}}{(n-1) !}\right)+\sqrt{-1 \partial}\left(\log u_{\epsilon} \frac{\partial \omega^{n-1}}{(n-1) !}\right) \\
= & \sqrt{-1} \partial\left(\bar{\partial} \log u_{\epsilon} \wedge \frac{\omega^{n-1}}{(n-1) !}\right)+\sqrt{-1 \partial}\left(\log \left(\frac{u}{\epsilon}+1\right) \frac{\partial \omega^{n-1}}{(n-1) !}\right) .
\end{aligned}
$$

Notice that

$$
\int_{M} \log \left(\frac{u}{\epsilon}+1\right) \frac{\omega^{n}}{n !} \leq \int_{M} \frac{u}{\epsilon} \frac{\omega^{n}}{n !}<\infty
$$

This together with (2.11), the condition that $\left|d\left(\omega^{n-1}\right)\right| \in L^{\infty}(M)$ and lemma 2.1, gives us that

$$
0=\lim _{i \rightarrow+\infty} \int_{B_{i}} \Delta \log u_{\epsilon}=\int_{M} \frac{f u}{u+\epsilon} \frac{\omega^{n}}{n !}+\int_{M} \frac{1}{u(u+\epsilon)^{2}}\left|d u^{2}\right| \frac{\omega^{n}}{n !}
$$

for all $\epsilon>0$, where $B_{i}^{\prime} s$ are the ones in the lemma 2.1. By the same argument in [18], we know that $u$ must be a constant. The proof of Theorem 1.2 is therefore completed.

\section{A vanishing theOrem on Higgs Bundle}

Let $(M, \omega)$ be an $n$-dimensional Hermitian manifold. A Higgs bundle $\left(E, \bar{\partial}_{E}, \theta\right)$ over $M$ is a holomorphic bundle $\left(E, \bar{\partial}_{E}\right)$ coupled with a Higgs field $\theta \in \Omega_{M}^{1,0}(\operatorname{End}(E))$ such that $\bar{\partial}_{E} \theta=0$ and $\theta \wedge \theta=0$. Higgs bundles first emerged thirty years ago in Hitchin's ([8]) study of the self duality equations on a Riemann surface and in Simpson's subsequent work ([15], [16]) on nonabelian Hodge theory. Such objects have rich structures and play an important role in many areas including gauge theory, Kähler and hyperkähler geometry, group representations and nonabelian Hodge theory. Let $H$ be a Hermitian metric on the bundle $E$, we consider the Hitchin-Simpson connection

$$
\bar{\partial}_{\theta}:=\bar{\partial}_{E}+\theta, \quad D_{H, \theta}^{1,0}:=D_{H}^{1,0}+\theta^{*_{H}}, \quad D_{H, \theta}=\bar{\partial}_{\theta}+D_{H, \theta}^{1,0},
$$


where $D_{H}$ is the Chern connection of $\left(E, \bar{\partial}_{E}, H\right)$ and $\theta^{*_{H}}$ is the adjoint of $\theta$ with respect to the metric $H$. The curvature of this connection is

$$
F_{H, \theta}=F_{H}+\left[\theta, \theta^{* H}\right]+\partial_{H} \theta+\bar{\partial}_{E} \theta^{*_{H}},
$$

where $F_{H}$ is the curvature of $D_{H}$ and $\partial_{H}$ is the $(1,0)$-part of $D_{H}$.

Let $s$ be a $\theta$-invariant holomorphic section of a Higgs bundle $\left(E, \bar{\partial}_{E}, \theta\right)$, i.e. there exists a holomorphic 1-form $\eta$ on $M$ such that $\theta(s)=\eta \otimes s$. When the base manifold $(M, \omega)$ is compact, following Kobayashi's techniques [9], one can obtain vanishing theorems for $\theta$-invariant holomorphic sections on Higgs bundles or Higgs sheaves (see [1, 3, 13]). Now we consider the case that the base manifold is complete non-Kähler.

Since $s$ is $\theta$-invariant, by the formula (4.50) in [13], we know

$$
\sqrt{-1} \Lambda_{\omega}\left\langle s,-\left[\theta, \theta^{* H}\right] s\right\rangle_{H}=\left|\theta^{* H} s-\left\langle\theta^{* H} s, s\right\rangle_{H} \frac{s}{|s|_{H}^{2}}\right|_{H, \omega}^{2} \geq 0
$$

A straightforward calculation shows the following Weitzenböck formula

$$
\begin{aligned}
& \sqrt{-1} \Lambda_{\omega} \partial \bar{\partial}|s|_{H}^{2} \\
= & \left|D_{H}^{1,0} s\right|_{H, \omega}^{2}+\sqrt{-1} \Lambda_{\omega}\left\langle s, F_{H} s\right\rangle_{H} \\
= & \left|D_{H}^{1,0} s\right|_{H, \omega}^{2}-\left\langle s, \sqrt{-1} \Lambda_{\omega} F_{H, \theta} s\right\rangle_{H}-\sqrt{-1} \Lambda_{\omega}\left\langle s,\left[\theta, \theta^{* H}\right] s\right\rangle_{H} \\
\geq & \left|D_{H}^{1,0} s\right|_{H, \omega}^{2}-\left\langle s, \sqrt{-1} \Lambda_{\omega} F_{H, \theta} s\right\rangle_{H}
\end{aligned}
$$

on $M$. On the other hand, it holds that

$$
\sqrt{-1} \Lambda_{\omega} \partial|s|_{H}^{2} \wedge \bar{\partial}|s|_{H}^{2} \leq|s|_{H}^{2}\left|D_{H}^{1,0} s\right|_{H}^{2}
$$

Then these yield that

$$
\begin{aligned}
& \sqrt{-1} \Lambda_{\omega} \partial \bar{\partial} \log |s|_{H}^{2} \\
= & \frac{1}{|s|_{H}^{2}} \sqrt{-1} \Lambda_{\omega} \partial \bar{\partial}|s|_{H}^{2}-\frac{1}{|s|_{H}^{4}} \sqrt{-1} \Lambda_{\omega} \partial|s|_{H}^{2} \wedge \bar{\partial}|s|_{H}^{2} \\
\geq & -\frac{1}{|s|_{H}^{2}}\left\langle s, \sqrt{-1} \Lambda_{\omega} F_{H, \theta} s\right\rangle_{H} .
\end{aligned}
$$

Together with Theorem 1.2, we conclude

Theorem 3.1. Let $(M, \omega)$ be a complete Gauduchon manifold of complex dimension $n$ with $\left|d\left(\omega^{n-1}\right)\right| \in L^{\infty}(M)$, and $\left(E, \bar{\partial}_{E}, \theta\right)$ be a Higgs bundle over $M$. If there exists a Hermitian metric $H$ on $E$ such that $\sqrt{-1} \Lambda_{\omega} F_{H, \theta} \leq-f I d_{E}$, where $f$ is a continuous function on $M$ which is bounded from below and $\int_{M} f \omega^{n}>0$. Then $E$ admits no non-zero $\theta$-invariant holomorphic $L^{p}$-sections for $0<p<\infty$.

\section{REFERENCES}

[1] U.Bruzzo and B.Graña Otero, Metrics on semistable and numerically effective Higgs bundles, J. Reine Angew. Math., 612(2007), 59-79.

[2] N.P. Buchdahl, Hermitian-Einstein connections and stable vector bundles over compact complex surfaces, Math. Ann., 280(1988), no. 4, 625-648. 
[3] S.A. Cardona, On vanishing theorems for Higgs bundles, Differential Geom. Appl., 35 (2014), 95-102.

[4] S.K. Donaldson, Anti self-dual Yang-Mills connections over complex algebraic surfaces and stable vector bundles, Proc. London Math. Soc., 50 (1985), no. 1, 1-26.

[5] M.P. Gaffney, A special Stokes's theorem for complete Riemannian manifolds, Ann. of Math.(2), 60 (1954), 140-145.

[6] P. Gauduchon, Torsion 1-forms of compact Hermitian manifolds, Math. Ann., 267 (1984), no. 4, 495-518.

[7] R. E. Greene and H. Wu, Curvature and complex analysis, Bull. Amer. Math. Soc., 77(1971), 10451049.

[8] N.J. Hitchin, The self-duality equations on a Riemann surface, Proc. London Math. Soc. (3), 55 (1987), no. 1, 59-126.

[9] S. Kobayashi, Curvature and stability of vector bundles, Proc. Japan Acad. Ser. A Math. Sci., 58 (1982), no. 4, 158-162.

[10] J. Li and S.T. Yau, Hermitian-Yang-Mills connection on non-Kähler manifolds, Mathematical aspects of string theory (San Diego, Calif., 1986), 560-573, Adv. Ser. Math. Phys., 1, World Sci. Publishing, Singapore, 1987.

[11] M. Lübke and A. Teleman, The Kobayashi-Hitchin correspondence, World Scientific Publishing Co., Inc., River Edge, NJ, 1995.

[12] M. Lübke and A. Teleman, The universal Kobayashi-Hitchin correspondence on Hermitian manifolds, Mem. Amer. Math. Soc., 183 (2006), no. 863.

[13] J.Y. Li, C. J. Zhang, X. Zhang, Semi-stable Higgs sheaves and Bogomolov type inequality, Calc. Var. Partial Differential Equations, 56 (2017), no. 3, Art. 81, 33 pp.

[14] M.S. Narasimhan and C.S. Seshadri, Stable and unitary vector bundles on a compact Riemann surface, Ann. of Math. (2), 82(1965), 540-567.

[15] C.T. Simpson, Constructing variations of Hodge structure using Yang-Mills theory and applications to uniformization, J. Amer. Math. Soc., 1 (1988), no. 4, 867-918.

[16] C.T. Simpson, Higgs bundles and local systems, Inst. Hautes Études Sci. Publ. Math., 75(1992), 5-95.

[17] K.K. Uhlenbeck and S. T. Yau, On the existence of Hermitian-Yang-Mills connections in stable vector bundles, Comm. Pure Appl. Math. 39(1986), no. S, suppl., S257-S293.

[18] S.T. Yau, Some function-theoretic properties of complete Riemannian manifold and their applications to geometry, Indiana Univ. Math. J., 25(1976), no. 7, 659-670.

Yuang Li, Chuanjing Zhang and Xi Zhang

School of Mathematical Sciences, University of Science and Technology of China

Anhui 230026, P.R. China

Email:lya1997@mail.ustc.edu.cn; chjzhang@mail.ustc.edu.cn; mathzx@ustc.edu.cn 